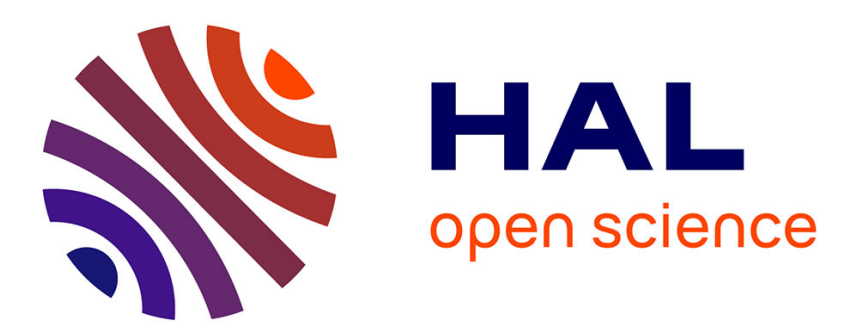

\title{
Fault estimation filter design for discrete-time descriptor systems
}

Zhenhua Wang, Mickael Rodrigues, Didier Theilliol, Yi Shen

\section{To cite this version:}

Zhenhua Wang, Mickael Rodrigues, Didier Theilliol, Yi Shen. Fault estimation filter design for discrete-time descriptor systems. IET Control Theory and Applications, 2015, 9 (10), pp.1587-1594. 10.1049/iet-cta.2014.0641 . hal-01133353

\section{HAL Id: hal-01133353 https://hal.science/hal-01133353}

Submitted on 19 Mar 2015

HAL is a multi-disciplinary open access archive for the deposit and dissemination of scientific research documents, whether they are published or not. The documents may come from teaching and research institutions in France or abroad, or from public or private research centers.
L'archive ouverte pluridisciplinaire $\mathbf{H A L}$, est destinée au dépôt et à la diffusion de documents scientifiques de niveau recherche, publiés ou non, émanant des établissements d'enseignement et de recherche français ou étrangers, des laboratoires publics ou privés. 


\title{
Fault estimation filter design for discrete-time descriptor systems
}

\author{
Zhenhua Wang ${ }^{\mathrm{a}}$, Mickael Rodrigues ${ }^{\mathrm{b}}$, Didier Theilliol*, c, Yi Shen ${ }^{\mathrm{a}}$ \\ ${ }^{a}$ School of Astronautics, Harbin Institute of Technology, Harbin, 150001, P. R. China \\ ${ }^{b}$ Laboratoire d'Automatique et Génie des Procédés, Université de Lyon, Lyon, F-69003, France \\ ${ }^{c}$ Centre de Recherche en Automatique de Nancy, Université de Lorraine, CNRS UMR 7039, F-54506 \\ Vandoeuvre-les-Nancy, France
}

\begin{abstract}
This paper deals with actuator fault estimation for discrete-time linear descriptor systems. The main contribution lies in the synthesis of a novel filter to estimate and isolate actuator faults for discrete linear descriptor systems. In this paper, a restricted system equivalent model is firstly obtained for the considered descriptor system, and then a fault estimation filter is designed based on the restricted system equivalent representation. The gain matrix of the proposed filter has a special structure such that the residual vector will tend to the actuator fault with certain time delays which equal to the fault detectability indexes. Morever, this paper proposes a robust filter design method to deal with the effect of the unknown disturbance. The proposed filter is able to detect and estimate the faults despite in the presence of disturbance. The design conditions for the proposed filter are formulated as a Linear Matrix Inequality (LMI) feasibility problem, which can be solved efficiently. Simulation results are given to illustrate the effectiveness of the proposed method.
\end{abstract}

Keywords: Actuator faults, Descriptor systems, Discrete-time, Fault estimation, Filter design, LMI

\footnotetext{
*Corresponding author. Tel: +3338368 65; fax: +33 3836844 62. E-mail: didier.theilliol@univ-lorraine.fr
} 


\section{Introduction}

The model-based fault diagnosis techniques have received considerable attention during the past decades. Various methods have been proposed in the literature, e.g. observer-based fault detection method $[1,2]$, parity space approach [3], $H_{\infty}$ fault detection filter $[4,5]$, and the recently presented finite frequency fault detection method [6-8]. For more comprehensive knowledge on model-based fault diagnosis techniques, see the monographs [9-11], survey papers [12-14] and references therein.

On another research front, descriptor systems appear in many fields such as power systems, electrical networks, and mechanical systems $[15,16]$. In the last few decades, many approaches have been proposed to design observers for descriptor systems [17-22]. References [17-19] have studied the observer design problem in linear descriptor systems. Observer design methods for nonlinear descriptor systems have been reported in [20-22].

Compared with the results on observer design for the fault-free descriptor systems, there are limited papers on fault diagnosis methods for descriptor systems [23-29]. [23] has proposed a fault estimation method for linear descriptor systems by using on-line learning methodology. In [23], the state variables are required to be fully measurable. However, in many situations, it is too expensive or even impossible to measure all the states. [24] has considered the robust fault detection problem for linear descriptor systems via generalized unknown input observers. Nevertheless, as pointed out in [24], these generalized observers are difficult to implement due to the fact that the derivatives of known signals may be involved in realizing the generalized observers. More recently, [25] has studied fault estimation and fault-tolerant control for descriptor systems by using proportional, multipleintegral and derivative observer. [26] has proposed an actuator fault estimation method for a class of nonlinear descriptor systems via neural adaptive observer design. It should be noticed that most of the aforementioned results focus on fault diagnosis in continuous-time descriptor systems, only [27-29] have considered fault diagnosis methods for discrete-time descriptor systems. In [27], 
an LMI-based unknown input observer design method is proposed and applied to fault detection and isolation for Takagi-Sugeno descriptor systems. [28] has proposed an $H_{-} / H_{\infty}$ fault detection filter for a class of discrete-time descriptor systems described by Linear Parameter-Varying (LPV) form with a globally Lipschitz term. However, the methods in [27] and [28] only address the fault detection problem but the fault magnitudes cannot be estimated. As pointed out in [30], accurate and timely fault estimation is an important basis for active control reconfiguration. For regular systems, several model-based techniques such as parity space approach, adaptive observer and sliding mode observer have been proposed to achieve fault estimation [31-36]. However, little work has been done to fault estimation for descriptor systems [25, 26, 29], especially for the discrete descriptor systems. In [29], a fault estimation method based on Proportional-Integral (PI) observer is proposed to deal with fault estimation for discrete-time LPV descriptor systems. However, the method in [29] requires the faults to be constant or slow varying, which is a restrictive condition.

In this paper, a novel fault estimation filter design method without the constant fault assumption is proposed for discrete-time linear descriptor systems. A restricted system equivalent model of the considered descriptor system is firstly established. We firstly construct a restricted system equivalent model of the considered descriptor system, and then design a fault estimation filter based on the restricted system equivalent representation. The filter design method is inspired by [37] such that the $i$ th component of the fault estimation vector asymptotically converges to the $i$ th fault with a time delay equals to its fault detectability index. Moreover, this paper proposes a robust filter design method to deal with the effect of the unknown disturbance, which is not considered in [37]. In this paper, design conditions for the proposed filter are formulated as a linear matrix inequality (LMI) feasibility problem, which can be easily solved by using standard LMI toolboxes. The main novelty of this paper lies on the following aspects. First, the proposed approach does not assume the faults to be constant. Therefore, it has a larger application scope 
than the method based on PI observers. Secondly, the form of the proposed filter is similar with the normal state observer, which is easy to implement. Moreover, this paper proposes a robust filter which is able to attenuate the effect of unknown disturbance and to provide a robust fault estimation. In addition, the proposed filter design method provides a dynamic detection threshold for the residuals in the fault-free situation. This can be used to achieve robust fault detection.

The rest of this paper is organized as follows. The problem formulation is presented in Section 2. The fault estimation filter is designed in Section 3. In Section 4, a numerical example is given to illustrate the effectiveness of the proposed approach. Conclusions are given in Section 5 .

\section{Problem statement and preliminaries}

Consider the following discrete-time descriptor system with actuator faults

$$
\left\{\begin{array}{l}
E x(k+1)=A x(k)+B u(k)+F n(k)+D w(k) \\
y(k)=C x(k)
\end{array}\right.
$$

where $x(k) \in \mathbb{R}^{n}$ is the state vector, $u(k) \in \mathbb{R}^{p}$ is the input vector, $w(k) \in \mathbb{R}^{d}$ denotes the unknown disturbance and $y(k) \in \mathbb{R}^{m}$ is the output vector. $E \in \mathbb{R}^{n \times n}$ may be rank deficient, i.e. $\operatorname{rank}(E)=r \leq n, A, B, C$ and $D$ are known matrices with appropriate dimensions. $n(k)=$ $\left[n_{1}(k) n_{2}(k) \cdots \quad n_{q}(k)\right]^{T} \in \mathbb{R}^{q}$ is the vector of fault magnitudes and $F=\left[\begin{array}{llll}f_{1} & f_{2} & \cdots & f_{q}\end{array}\right] \in \mathbb{R}^{n \times q}$ is the fault distribution matrix. In the following of the paper, we assume that $\operatorname{rank}(C)=m$, $\operatorname{rank}(F)=q, q \leq m$, and

$$
\operatorname{rank}\left[\begin{array}{c}
E \\
C
\end{array}\right]=n
$$

In this paper, a filter is designed to detect and estimate the faults in descriptor system (1). To this end, some usefull preliminaries are presented in this section. First, recall the definition of restricted system equivalent. 
Definition 1 [15]. The following descriptor systems

$$
\left\{\begin{array}{l}
E x(k+1)=A x(k)+B u(k) \\
y(k)=C x(k)
\end{array}\right.
$$

and

$$
\left\{\begin{array}{l}
\tilde{E} \tilde{x}(k+1)=\tilde{A} \tilde{x}(k)+\tilde{B} u(k) \\
y(k)=\tilde{C} \tilde{x}(k)
\end{array}\right.
$$

are called Restricted System Equivalent (RSE) if there exist two nonsingular matrices $Q$ and $P$ such that

$$
x(k)=P \tilde{x}(k)
$$

and

$$
Q E P=\tilde{E}, Q A P=\tilde{A}, Q B=\tilde{B}, C P=\tilde{C}
$$

In this paper, it is required that $T \in \mathbb{R}^{n \times n}$ is a nonsingular matrix satisfing the following equiation

$$
T E+N C=I_{n}
$$

where $N \in \mathbb{R}^{n \times m}$ is an arbitrary matrix.

In order to ensure the existence of such a matrix $T$, the following Lemma is introduced.

Lemma 1. Under the assumption (2), there exist a nonsingular matrix $T \in \mathbb{R}^{n \times n}$ and a matrix $N \in \mathbb{R}^{n \times m}$ satisfying

$$
T E+N C=I_{n}
$$

Proof. Since $\operatorname{rank}(E)=r$, there exist two nonsingular matrices $Q_{0}$ and $P_{0}$ such that

$$
\begin{aligned}
& {\left[\begin{array}{c}
x_{1}(k) \\
x_{2}(k)
\end{array}\right]=P_{0}^{-1} x(k), Q_{0} E P_{0}=\left[\begin{array}{cc}
I_{r} & 0 \\
0 & 0
\end{array}\right], Q_{0} A P_{0}=\left[\begin{array}{cc}
A_{11} & A_{12} \\
A_{21} & A_{22}
\end{array}\right],} \\
& Q_{0} B=\left[\begin{array}{c}
B_{1} \\
B_{2}
\end{array}\right], Q_{0} F=\left[\begin{array}{c}
F_{1} \\
F_{2}
\end{array}\right], Q_{0} D=\left[\begin{array}{c}
D_{1} \\
D_{2}
\end{array}\right], C P_{0}=\left[\begin{array}{ll}
C_{1} & C_{2}
\end{array}\right]
\end{aligned}
$$


then the system (1) is restricted system equivalent to

$$
\left\{\begin{array}{l}
Q_{0} E P_{0}\left[\begin{array}{c}
x_{1}(k+1) \\
x_{2}(k+1)
\end{array}\right]=Q_{0} A P_{0}\left[\begin{array}{l}
x_{1}(k) \\
x_{2}(k)
\end{array}\right]+Q_{0} B u(k)+Q_{0} F n(k)+Q_{0} D w(k) \\
y(k)=C P_{0}\left[\begin{array}{l}
x_{1}(k) \\
x_{2}(k)
\end{array}\right]
\end{array}\right.
$$

Under the assumption (2), it is easy to shown that

$$
\operatorname{rank}\left[\begin{array}{c}
Q_{0} E P_{0} \\
C P_{0}
\end{array}\right]=\operatorname{rank}\left[\begin{array}{cc}
I_{r} & 0 \\
0 & 0 \\
C_{1} & C_{2}
\end{array}\right]=\operatorname{rank}\left[\begin{array}{cc}
I_{r} & 0 \\
C_{1} & C_{2}
\end{array}\right]=n
$$

Let $H=\left[\begin{array}{ll}0 & I_{m}\end{array}\right]^{T}$, we have

$$
Q_{0} E P_{0}+H C P_{0}=\left[\begin{array}{cc}
I_{r} & 0 \\
C_{1} & C_{2}
\end{array}\right]
$$

Since $P_{0}$ is nonsingular, the following matrix

$$
G \triangleq Q_{0} E+H C=\left[\begin{array}{cc}
I_{r} & 0 \\
C_{1} & C_{2}
\end{array}\right] P_{0}^{-1}
$$

is also a nonsingular matrix.

Let

$$
T=G^{-1} Q_{0}, N=G^{-1} H
$$

we get a nonsingular matrix $T$ and a matrix $N$ such that (8) holds.

Remark 1. Lemma 1 guarantees the existence of a nonsingular matrix $T$ which also satisfies the constraint (8). However, it is not very computationally efficient. Therefore, the following Lemma is introduced to provide a convenient alternative to find such a matrix $T$.

Lemma 2 [38]. Given matrices $\mathcal{X} \in \mathbb{R}^{a \times b}, \mathcal{Y} \in \mathbb{R}^{b \times c}$, and $\mathcal{Z} \in \mathbb{R}^{a \times c}$. If $\mathcal{Y}=c$, the general solution of $\mathcal{X Y}=\mathcal{Z}$ is given by

$$
\mathcal{X}=\mathcal{Z} \mathcal{Y}^{\dagger}+\mathcal{S}\left[I_{b}-\mathcal{Y} \mathcal{Y}^{\dagger}\right]
$$


where $\mathcal{S} \in \mathbb{R}^{a \times b}$ is an arbitrary matrix. Herein and throughout this paper, the symbol ${ }^{\dagger}$ is use to denote the pseudo-inverse of a matrix.

Since rank $\left[\begin{array}{c}E \\ C\end{array}\right]=n$, there exist matrices $T$ and $N$ satisfying (7). Using Lemma 2, the general solution for $T$ and $N$ is given by

$$
T=\mathcal{M R}_{T}, N=\mathcal{M R}_{N}
$$

where

$$
\mathcal{M}=\left[\begin{array}{c}
E \\
C
\end{array}\right]^{\dagger}+S\left(I_{n+m}-\left[\begin{array}{c}
E \\
C
\end{array}\right]\left[\begin{array}{c}
E \\
C
\end{array}\right]^{\dagger}\right), \mathcal{R}_{T}=\left[\begin{array}{c}
I_{n} \\
0
\end{array}\right], \mathcal{R}_{N}=\left[\begin{array}{c}
0 \\
I_{m}
\end{array}\right]
$$

and $S \mathbb{R}^{n \times(n+m)}$ is an arbitrary matrix, which provides us the degrees of design freedom. By choosing matrix $S$, we can determine a nonsingular matrix $T$ which satisfies equation (7).

Since $T$ is a nonsingular matrix, descriptor system (1) is RSE to the following representation

$$
\left\{\begin{array}{l}
T E x(k+1)=T A x(k)+T B u(k)+T F n(k)+T D w(k) \\
y(k)=C x(k)
\end{array}\right.
$$

Motivated by [37], this paper introduces the following definitions of fault detectability indexes and fault detectability matrix, which will be used in the sequel.

Definition 2. The fault detectability indexes of system (18) are defined as $\rho=\left\{\rho_{1}, \rho_{2}, \cdots, \rho_{q}\right\}$ where

$$
\rho_{i}=\min \left\{v: C(T A)^{v-1} T f_{i} \neq 0, v=1,2, \cdots\right\}
$$

Without loss of generality, it is assumed that the system (18) has finite fault detectability indexes and $s \triangleq \min \left\{\rho_{1}, \rho_{2}, \cdots, \rho_{q}\right\}$ is used to represent the maximum value of fault detectability indexes.

Definition 3. With the assumption that the system (18) has finite fault detectability indexes, 
the fault detectability matrix is defined as

$$
\Psi=\left[\begin{array}{llll}
(T A)^{\rho_{1}-1} T f_{1} & (T A)^{\rho_{2}-1} T f_{2} & \cdots & (T A)^{\rho_{q}-1} T f_{q}
\end{array}\right\}
$$

Besides, the discrete-time Bounded Real Lemma will be used in this paper.

Lemma 3 [40]. Consider the state-space system

$$
\left\{\begin{array}{l}
x(k+1)=A x(k)+B w(k) \\
y(k)=C x(k)+D w(k)
\end{array}\right.
$$

For a given scalar $\gamma>0$, the following statements are equivalent:

(i) $A$ is a stable matrix and $\left\|C(z I-A)^{-1} B+D\right\|<\gamma$.

(ii) There exists a symmetric $P$ satisfying $P>0$ and the following matrix inequality

$$
\left[\begin{array}{cc}
A^{T} P A-P+C^{T} C & A^{T} P B+C^{T} D \\
B^{T} P A+D^{T} C & -\gamma^{2} I+B^{T} P B+D^{T} D
\end{array}\right]<0
$$

\section{Fault estimation filter design}

Based on the RSE representation (18), the following filter is constructed for descriptor system (1)

$$
\left\{\begin{array}{l}
z(k+1)=T A \hat{x}(k)+T B u(k)+L(y(k)-C \hat{x}(k)) \\
\hat{x}(k)=z(k)+N y(k) \\
r(k)=W(y(k)-C \hat{x}(k))
\end{array}\right.
$$

where $z(k) \in \mathbb{R}^{n}$ is an intermediate state vector, $\hat{x}(k) \in \mathbb{R}^{n}$ is the state estimation vector and $r(k) \in \mathbb{R}^{q}$ denotes the residual vector. In (23), matrices $T$ and $N$ can be determined by using (16), $L \in \mathbb{R}^{n \times m}$ and $W \in \mathbb{R}^{q \times m}$ are matrices to be designed.

Define the state estimation error and output estimation error as

$$
e(k)=x(k)-\hat{x}(k)
$$


and

$$
\epsilon(k)=y(k)-C \hat{x}(k)
$$

respectively. Then, by using (7), (18) and (23), the error dynamic system is obtained as

$$
\left\{\begin{array}{l}
e(k+1)=(T A-L C) e(k)+T F n(k)+T D w(k) \\
\epsilon(k)=C e(k)
\end{array}\right.
$$

In order to analyse the estimation error, the error system (26) is split into two parts, i.e.

$$
\left\{\begin{array}{l}
e_{f}(k+1)=(T A-L C) e_{f}(k)+T F n(k) \\
\epsilon_{f}(k)=C e_{f}(k)
\end{array}\right.
$$

and

$$
\left\{\begin{array}{l}
e_{w}(k+1)=(T A-L C) e_{w}(k)+T D w(k) \\
\epsilon_{w}(k)=C e_{w}(k)
\end{array}\right.
$$

where

$$
\begin{gathered}
e(k)=e_{f}(k)+e_{w}(k) \\
\epsilon(k)=\epsilon_{f}(k)+\epsilon_{w}(k) \\
\epsilon_{f}(k)=C e_{f}(k), \epsilon_{w}(k)=C e_{w}(k)
\end{gathered}
$$

Remark 2. The idea of separating (26) as (27) and (28) is inspired by [39], where a very similar methodology was used to analyze and design an adaptive observer. With this treatment, the output estimation error $\epsilon(k)$ is split into two components, $\epsilon_{f}(k)$ and $\epsilon_{w}(k)$. The first component $\epsilon_{f}(k)$ reflects the impact of fault on the output estimation error, while the other part $\epsilon_{w}(k)$ contains the influence of the disturbance. Note that this separation is only used to facilitate the analysis of error dynamic (26) and the design of filter (23). It should be noticed that only the out estimation error $\epsilon(k)$, which is available in practice, will be used in the proposed fault estimation method. Therefore, this separation does not involve any practical realization problem. 
Then, the following Theorem is proposed to constrain the structure of matrix $L$ in the filter (23). This Theorem is of essential importance in filter design.

Theorem 1. If there exists a matrix $L$ satisfying

$$
(T A-L C) \Psi=0
$$

then $\epsilon_{f}(k)$ can be expressed as

$$
\epsilon_{f}(k)=C(T A-L C)^{k} e(0)+\Upsilon\left[n_{1}\left(k-\rho_{1}\right) n_{2}\left(k-\rho_{2}\right) \cdots n_{q}\left(k-\rho_{q}\right)\right]^{T}, k>k_{f}+s
$$

where $\Upsilon=C \Psi, e(0)$ is the initial estimation error and $k_{f}$ denotes the time instant when faults occur.

Proof. From (27), it is easy to derive that

$$
\begin{aligned}
\epsilon_{f}(k)= & C(T A-L C) e_{f}(k-1)+C T F n(k-1) \\
= & C(T A-L C)^{2} e_{f}(k-2)+C(T A-L C) T F n(k-2)+C T F n(k-1) \\
& \vdots \\
= & C(T A-L C)^{k} e_{f}(0)+C G_{k-k_{f}} T F n\left(k_{f}\right)+\cdots+C G_{s} T F n(k-s)+\cdots+C G_{1} T F n(k-1)
\end{aligned}
$$

where $k>s+k_{f}$, matrix $G_{j}$ is given by

$$
G_{j}=(T A-L C)^{j-1}, j=1, \cdots, k-k_{f}
$$

From the definitions of $T$ and $n(k)$, we have

$$
C G_{j} T F n(k-j)=C G_{j} T f_{1} n_{1}(k-j)+\cdots+C G_{j} T f_{i} n_{i}(k-j)+\cdots+C G_{j} T f_{q} n_{q}(k-j)
$$

Now, we should discuss about $C G_{j} T f_{i}$. By using the definition of fault detectability indexes, we have

$$
\begin{gathered}
C G_{j} T f_{i}=C(T A)^{j-1} T f_{i}=0, j<\rho_{i} \\
C G_{j} T f_{i}=C(T A)^{\rho_{i}-1} T f_{i}, j=\rho_{i}
\end{gathered}
$$




$$
C G_{j} T f_{i}=C(T A-L C)^{j-\rho_{i}}(T A)^{\rho_{i}-1} T f_{i}=0, j>\rho_{i}
$$

Using (32), we obtain

$$
(T A-L C)(T A)^{\rho_{i}-1} T f_{i}=0
$$

Substituting (40) into (39) yields

$$
C G_{j} T f_{i}=0, j>\rho_{i}
$$

To sum up, we have

$$
C G_{j} T f_{i}=\left\{\begin{array}{ccc}
C(T A)^{\rho_{i}-1} T f_{i} & j=\rho_{i} \\
0 & j \neq \rho_{i} &
\end{array} \quad j=1, \cdots, k-k_{f}\right.
$$

It follows that

$$
C G_{j} T f_{i}=\left\{\begin{array}{ccc}
C(T A)^{\rho_{i}-1} T f_{i} & j=\rho_{i} \\
0 & j \neq \rho_{i} &
\end{array} \quad j=1, \cdots, k-k_{f}\right.
$$

Substituting (43) into (34) gives

$$
\begin{aligned}
\epsilon_{f}(k) & =C(T A-L C)^{k} e(0)+C(T A)^{\rho_{q}-1} T f_{q} n_{q}\left(k-\rho_{q}\right)+\cdots+C(T A)^{\rho_{1}-1} T f_{1} n_{1}\left(k-\rho_{1}\right) \\
& =C(T A-L C)^{k} e(0)+\Upsilon\left[n_{1}\left(k-\rho_{1}\right) n_{2}\left(k-\rho_{2}\right) \cdots n_{q}\left(k-\rho_{q}\right)\right]^{T}
\end{aligned}
$$

where $k>s+k_{f}$. This completes the proof.

Theorem 1 implies that the vector $\epsilon_{f}(k)$ will exhibit the form of (33) if the matrix $L$ satisfies the equation (32). Since the output estimation error $\epsilon(k)$ is composed of $\epsilon_{f}(k)$ and $\epsilon_{w}(k)$, the effect of $w(k)$ should be considered in the design of matrix $L$.

Based on Theorem 1, the following Theorem is proposed to design the fault estimation filter (23). First, the gain matrix $L$ in $(23)$ is required to have a special structure which makes the equation (32) satisfied. Moreover, the matrix $L$ is designed so that the residual $r(k)$ is robust against the disturbance $w(k)$.

Theorem 2. For a given scalar $\gamma>0$, if the following conditions are satisfied:

(i) $\operatorname{rank}(\Upsilon)=q$ 
(ii) there exist a symmetric positive definite matrix $P>0, P \in \mathbb{R}^{n \times n}$ and a matrix $Y \in \mathbb{R}^{n \times m}$ such that

$$
\left[\begin{array}{ccc}
-P+\left(\Upsilon^{\dagger} C\right)^{T}\left(\Upsilon^{\dagger} C\right) & 0 & A_{1}^{T} P-C_{1}^{T} Y^{T} \\
* & -\gamma^{2} I_{d} & (T D)^{T} P \\
* & * & -P
\end{array}\right]<0
$$

where

$$
A_{1}=T A-T A \Psi \Upsilon^{\dagger} C, C_{1}=\left(I_{m}-\Upsilon \Upsilon^{\dagger}\right) C
$$

Then, by choosing

$$
W=\Upsilon^{\dagger}
$$

and

$$
L=T A \Psi \Upsilon^{\dagger}-P^{-1} Y\left(I_{m}-\Upsilon \Upsilon^{\dagger}\right)
$$

the residual $r(k)$ in the filter $(23)$ satisfies the following robust performance

$$
\|\xi\| \leq \sqrt{\gamma^{2}\|w\|^{2}+\mathcal{N}(e(0), k)}
$$

where

$$
\begin{gathered}
\xi(k)=r(k)-n_{\mathrm{d}}(k) \\
n_{\mathrm{d}}(k) \triangleq\left[n_{1}\left(k-\rho_{1}\right) n_{2}\left(k-\rho_{2}\right) \cdots n_{q}\left(k-\rho_{q}\right)\right]^{T}
\end{gathered}
$$

and $\mathcal{N}(e(0), k)$ is a function satisfying $\lim _{k \rightarrow \infty} \mathcal{N}(e(0), k)=0$.

Proof. If $\operatorname{rank}(\Upsilon)=q$, then we have

$$
\Upsilon^{\dagger} \Upsilon=I_{q}
$$


By letting $W=\Psi^{\dagger}$ and considering (33), the residual $r(k)$ is given by

$$
\begin{aligned}
& r(k)=W \epsilon(k) \\
& =\Upsilon^{\dagger} \epsilon_{f}(k)+\Upsilon^{\dagger} \epsilon_{w}(k) \\
& =\Upsilon^{\dagger} C(T A-L C)^{k} e(0)+\left[n_{1}\left(k-\rho_{1}\right) n_{2}\left(k-\rho_{2}\right) \cdots n_{q}\left(k-\rho_{q}\right)\right]^{T}+\Upsilon^{\dagger} \epsilon_{w}(k) \\
& =\Upsilon^{\dagger} C(T A-L C)^{k} e(0)+n_{\mathrm{d}}(k)+\Upsilon^{\dagger} \epsilon_{w}(k)
\end{aligned}
$$

It follows that

$$
\xi(k)=\xi_{0}(k)-\xi_{w}(k)
$$

where

$$
\xi_{0}(k)=\Upsilon^{\dagger} C(T A-L C)^{k} e(0), \xi_{w}(k)=\Upsilon^{\dagger} \epsilon_{w}(k)
$$

Using triangle inequality gives

$$
\|\xi\|=\sqrt{\left\|\xi_{0}\right\|^{2}+\left\|\xi_{w}\right\|^{2}} \leq \sqrt{\left(\left\|\Upsilon^{\dagger} C\right\|\|(T A-L C)\|^{k}\|e(0)\|\right)^{2}+\left\|\xi_{w}\right\|^{2}}
$$

Let

$$
\mathcal{N}(e(0), k)=\left(\left\|\Upsilon^{\dagger} C\right\|\|(T A-L C)\|^{k}\|e(0)\|\right)^{2}
$$

It is obvious that $\mathcal{N}(e(0), k)$ satisfies $\lim _{k \rightarrow \infty} \mathcal{N}(e(0), k)=0$ if matrix $T A-L C$ is stable, i.e. $\|(T A-$ $L C) \|<1$ Moreover, the inequality (49) is fulfilled if $\left\|\xi_{w}\right\|<\|w\|$ holds.

Now, it is ready to show that $T A-L C$ is stable and $\left\|\xi_{w}\right\|<\|w\|$ holds if (45) is satisfied.

Applying Lemma 3 to system (28), it is obtained that matrix $T A-L C$ is stable and $\left\|\xi_{w}\right\|<\|w\|$ holds if the following matrix inequality is satisfied

$$
\left[\begin{array}{cc}
(T A-L C)^{T} P(T A-L C)-P+\left(\Upsilon^{\dagger} C\right)^{T}\left(\Upsilon^{\dagger} C\right) & (T A-L C)^{T} P D \\
* & -\gamma^{2} I+(T D)^{T} P T D
\end{array}\right]<0
$$

Using the Schur complement Lemma [41], the inequality (58) is equivalent to

$$
\left[\begin{array}{ccc}
-P+\left(\Upsilon^{\dagger} C\right)^{T}\left(\Upsilon^{\dagger} C\right) & 0 & (T A-L C)^{T} P \\
* & -\gamma^{2} I_{d} & (T D)^{T} P \\
* & * & -P
\end{array}\right]<0
$$


Since $\operatorname{rank}(\Upsilon)=q$, we can apply Lemma 2 to (32), then the general solution for $L$ is obtained as

$$
L=T A \Psi \Upsilon^{\dagger}+R\left(I_{m}-\Upsilon \Upsilon^{\dagger}\right)
$$

where $R \in \mathbb{R}^{n \times m}$ is an arbitrary matrix.

Substituting (60) into $T A-L C$ gives

$$
T A-L C=T A-T A \Psi \Upsilon^{\dagger}-R\left(I_{m}-\Upsilon \Upsilon^{\dagger}\right)=A_{1}-R C_{1}
$$

It follows that inequality (59) is equivalent to

$$
\left[\begin{array}{ccc}
-P+\left(\Upsilon^{\dagger} C\right)^{T}\left(\Upsilon^{\dagger} C\right) & 0 & \left(A_{1}-R C_{1}\right)^{T} P \\
* & -\gamma^{2} I_{d} & (T D)^{T} P \\
* & * & -P
\end{array}\right]<0
$$

It is obvious that inequality (62) becomes (45) by letting $Y=P R$. Now, it has been shown that $T A-L C$ is stable and $\left\|\xi_{w}\right\|<\|w\|$ holds if (45) is satisfied.

If there exist a symmetric positive definite matrix $P$ and a matrix $Y$ such that (45) holds. Then $R$ is given by

$$
R=P^{-1} Y
$$

Substituting (63) into (60) yields (48). This completes the proof.

Remark 3. It is known that fault detection is an essential task in fault diagnosis. To achieve the fault detection goal, a residual evaluation procedure is necessary. A commonly used strategy is the so-called norm based residual evaluation [10], i.e. the norm of the residual $r(k)$ is compared with a threshold to detect the occurrence of a fault. In this paper, the robust performance criterion (49) can be used to compute the detection threshold. Considering the fault-free situation $(n(k)=0)$ and using (49), it comes

$$
\|r\|=\|\xi\| \leq \sqrt{\gamma^{2}\|w\|^{2}+\mathcal{N}(e(0), k)}
$$


Without loss of generality, it is assumed that the bounds of $\|w\|$ and $\|e(0)\|$ are $\bar{w}$ and $\bar{e}_{0}$, respectively. Then, we can obtain the following fault detection scheme

$$
\begin{cases}\text { Fault }- \text { free } & \text { if }\|r\| \neq \theta(k) \\ \text { Fauly } & \text { if }\|r\|>\theta(k)\end{cases}
$$

where

$$
\theta(k)=\sqrt{\gamma^{2} \bar{w}^{2}+\mathcal{N}\left(\bar{e}_{0}, k\right)}
$$

is a dynamic detection threshold.

Remark 4. After the fault is detected, fault isolation and estimation should be implemented in order to obtain further information about the fault. It is noted that the proposed method is able to achieve fault isolation and estimation simultaneously. Note that the performance criterion in (49) implies that the residual $r(k)$ robustly tends to the delayed fault magnitudes vector $n_{\mathrm{d}}(k)$. First of all, the residual $r(k)$ can be viewed as an estimate of fault $n(k)$. Therefore, the fault estimation goal is achieved. From the point of view of fault isolation, the $i$ th component $r_{i}(k)$ of the residual can be used as a fault indicator for the $i$ th actuator because $r_{i}(k)$ robustly tends to the $i$ th fault $n_{i}\left(k-\rho_{i}\right)$ without affecting by other faults. In other words, the $i$ th residual component $r_{i}(k)$ can be used to determine whether the $i$ th actuator is fault.

Finally, for the sake of readability, the overall procedure for the proposed fault estimation filter design method is summarized in Algorithm 1.

\section{Simulations}

A simulation example is given in this section to demonstrate the design procedure and the effectiveness of the proposed fault estimation filter. 


\section{Algorithm 1 Fault estimation filter design for discrete-time descriptor systems}

Given the parameters of the descriptor system (1): $E, A, B, F, D, C$

Choose a matrix $S$

Solve the equation (7) and obtain a matrix $\mathcal{M}$

$\triangleright$ To design matrices $T$ and $N$

$T \leftarrow \mathcal{M} \mathcal{R}_{T}, N \leftarrow \mathcal{M} \mathcal{R}_{N}$

Calculate the fault detectability indexes: $\rho_{i} \leftarrow \min \left\{v: C(T A)^{v-1} T f_{i} \neq 0, v=1,2, \cdots\right\}$

Calculate the fault detectability matrix: $\Psi \leftarrow\left[\begin{array}{lll}(T A)^{\rho_{1}-1} T f_{1} & \cdots(T A)^{\rho_{q}-1} T f_{q}\end{array}\right]$

$\Upsilon \leftarrow C \Psi$

$\gamma \leftarrow \gamma_{0}$

repeat

Solve the LMI problem (45)

$\triangleright$ To design the gain matrix $L$

if the LMI (45) has a solution

$$
R \leftarrow P^{-1} Y
$$

else

$$
\gamma \leftarrow \gamma_{0}+\Delta \gamma
$$

end if

until the LMI (45) is solved

$$
\begin{aligned}
& L \leftarrow T A \Psi \Upsilon^{\dagger}-R\left(I_{m}-\Upsilon \Upsilon^{\dagger}\right) \\
& W \leftarrow \Upsilon^{\dagger}
\end{aligned}
$$

$\triangleright$ To obtain the matrix $W$ 
Suppose that the matrices of system (1) are given by

$$
\begin{gathered}
E=\left[\begin{array}{cccc}
1 & 0 & 0 & 0 \\
0 & 1 & 0 & 0 \\
0 & 0 & 1 & 0 \\
0 & 0 & 0 & 0
\end{array}\right], A=\left[\begin{array}{cccc}
0.9 & 0.005 & -0.095 & 0 \\
0.005 & 0.995 & 0.0997 & 0 \\
0.095 & -0.0997 & 0.99 & 0 \\
1 & 0 & 1 & 1
\end{array}\right] \\
B=F=\left[\begin{array}{ccc}
0.1 & 0 \\
1 & 1 \\
-0.1 & 1 \\
-1 & 0
\end{array}\right], D=\left[\begin{array}{c}
1 \\
1 \\
1 \\
0
\end{array}\right], C=\left[\begin{array}{cccc}
0 & 1 & 0 & 0 \\
0 & 0 & 1 & 0 \\
0 & 0 & 0 & 1
\end{array}\right]
\end{gathered}
$$

By choosing $S$ in (16) as

$$
S=\left[\begin{array}{lllllll}
1 & 0 & 0 & 0 & 0 & 0 & 0 \\
0 & 1 & 0 & 0 & 1 & 0 & 0 \\
0 & 0 & 1 & 0 & 0 & 1 & 0 \\
0 & 0 & 0 & 1 & 0 & 0 & 1
\end{array}\right]
$$

It comes the following matrices

$$
T=\left[\begin{array}{cccc}
1 & 0 & 0 & 0 \\
0 & 0.5 & 0 & 0 \\
0 & 0 & 0.5 & 0 \\
0 & 0 & 0 & 1
\end{array}\right], N=\left[\begin{array}{ccc}
0 & 0 & 0 \\
0.5 & 0 & 0 \\
0 & 0.5 & 0 \\
0 & 0 & 1
\end{array}\right]
$$

Note that $C T f_{1} \neq 0$ and $C T f_{2} \neq 0$, the fault detectability indexes are $\rho_{1}=1$ and $\rho_{2}=1$.

Then

$$
\Psi=\left[T f_{1} T f_{2}\right]=\left[\begin{array}{cc}
0.9 & 0 \\
0.0025 & 0.5 \\
0.0475 & 0.5 \\
1 & 0
\end{array}\right], \Upsilon=C \Psi=\left[\begin{array}{cc}
0.0025 & 0.5 \\
0.0475 & 0.5 \\
1 & 0
\end{array}\right]
$$


It is obvious that $\operatorname{rank}(\Upsilon)=2$, i.e. the condition (i) in Theorem 2 is satisfied.

By the definitions of matrices $A_{1}$ and $C_{1}$ given in Theorem 2, it follows

$$
A_{1}=\left[\begin{array}{cccc}
0.9 & 0.0682 & -0.0682 & -0.8069 \\
0.0025 & 0.2236 & -0.2235 & 0.0078 \\
0.0475 & -0.2712 & 0.2712 & -0.055 \\
1 & -0.4578 & 0.4568 & -0.9206
\end{array}\right], C_{1}=\left[\begin{array}{cccc}
0 & 0.4995 & -0.4995 & -0.0225 \\
0 & -0.4995 & 0.4995 & -0.0225 \\
0 & 0.0225 & -0.0225 & 0.001
\end{array}\right]
$$

By repeated applications of Theorem 2, we obtain a minimal $\gamma=1.5$ and the corresponding $L$ is

$$
L=\left[\begin{array}{ccc}
2.4511 & -2.4511 & 1.2553 \\
0.5068 & 0.0405 & 0.0049 \\
0.0814 & 0.3637 & 0.0675 \\
2.7122 & -1.7122 & 2.3917
\end{array}\right]
$$

In the following simulation, the input signal is $u(k)=[2 \sin (k) 2 \sin (k)]^{T}$, the initial value of state vector is $x(0)=\left[\begin{array}{llll}0.5 & 1 & 0 & -0.5\end{array}\right]^{T}$, while the initial state estimation is $\hat{x}\left(\begin{array}{l}0 \\ \text { ) }\end{array}=\left[\begin{array}{llll}0 & 1 & 0 & -0.5\end{array}\right]^{T}\right.$. The disturbance $w(k)$ in the simulation is set as a random noise drawn from the uniform distribution on the interval $(0,0.1)$. The reason to choose a random noise with uniform distribution is that this kind of noise exhibits both bias and stochastic properties. Herein, it is assumed that we can obtain the following priopri: $\bar{w}=0.11, \bar{x}_{0}=0.55$.

Consider the following actuator faults

$$
n_{1}(k)=\left\{\begin{array}{ll}
0 & k<80 \\
1 & k \geq 80
\end{array}, n_{2}(k)= \begin{cases}0 & k<100 \\
1.2 \sin (0.1 k) & k \geq 100\end{cases}\right.
$$

In this situation, the fault detection result is shown in Fig. 1. The threshold $\theta(k)$ is computed by (66). It can be seen that the obtained dynamic threshold $\theta(k)$ is able to quickly and accurately detect the occurrence of fault despite the presence of disturbance. The actuator faults and their fault estimations are depicted in Fig. 2. If there was no disturbance, the residual $r(k)$ should 
converge to the actuator $n(k)$ only one sample of delay since the fault detectability indexes are $\rho_{1}=1$ and $\rho_{2}=1$. In the presence of disturbances, the residual $r(k)$ still closely approximates the actuator fault $n(k)$, as shown in Fig. 2.

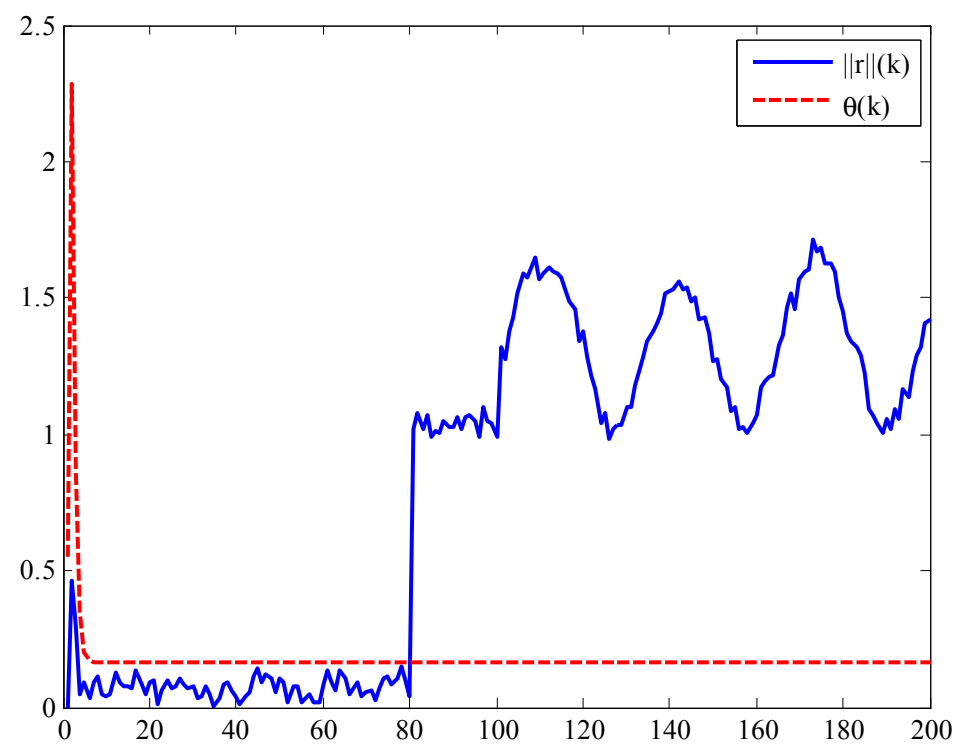

Figure 1: The fault detection result of the proposed filter

For comparative purpose, a PI observer proposed in [29] has been designed and simulated. Fig. 3 shows the actuator fault estimation results of the PI observer. As shown in Fig. 3, periodic vibration appears in the fault estimation of $n_{1}(k)$. This phenomenon illustrates that the fault estimation of $n_{1}(k)$ is disturbed by the dynamic of $n_{2}(k)$. In fact, the dynamics of fault estimations and state estimations vectors are coupled in the PI observer. Therefore, the fault estimation errors and state estimation errors are affected by each other and deteriorate the fault estimation performance. Moreover, it can be seen from Fig. 2 and Fig. 3 that our presented filter has faster convergence speed and better estimation accuracy than the PI observer from [29]. 

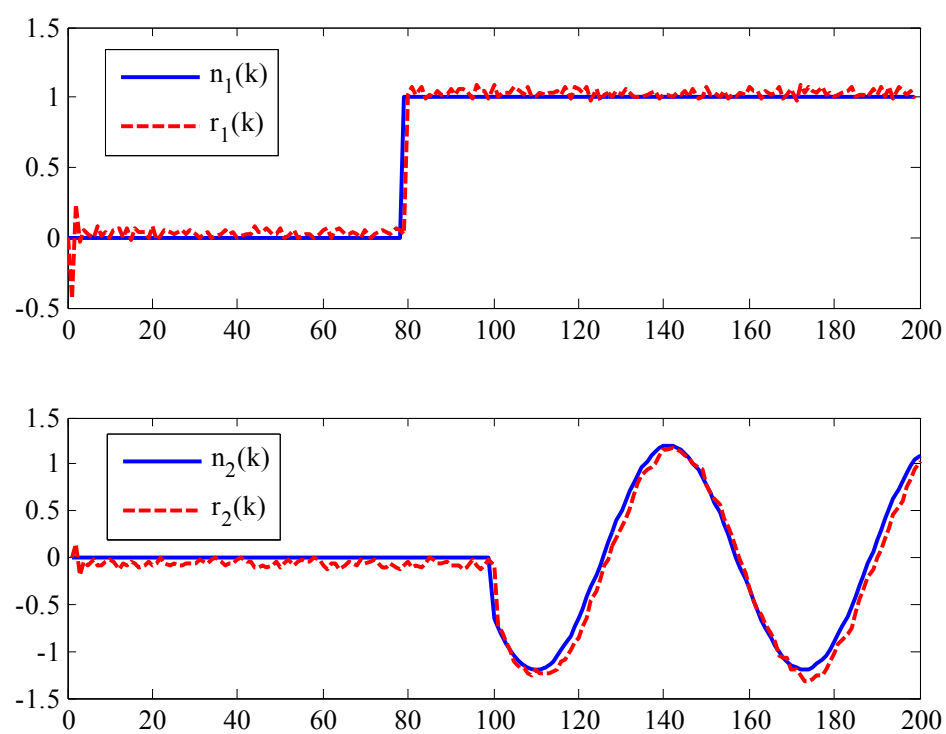

Figure 2: The fault estimation result of the proposed filter
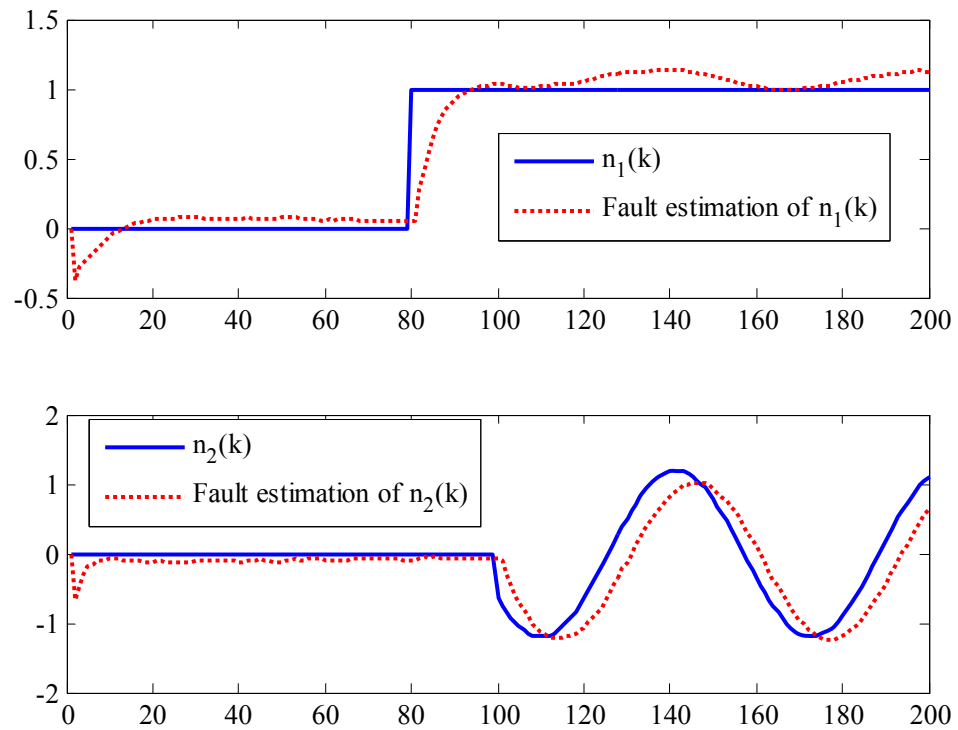

Figure 3: The fault estimation result of PI observer in [29] 


\section{Conclusion}

This paper proposes an actuator fault estimation filter design method for the discrete-time linear descriptor systems. The proposed fault estimation filter is able to detect and estimate actuator faults. Moreover, the filter is designed such that the residual is robust against the unknown disturbance. The filter design conditions have been derived as an LMI feasibility problem, which can be solved efficiently.

\section{Acknowledgements}

This work was financially supported by the National Natural Science Foundation of China (Grant No. 61273162). The authors would like to thank the associate editor and the anonymous reviewers for their helpful remarks, which have improved the quality of this paper.

\section{References}

[1] Seliger, R. and Frank, P. M.: Fault diagnosis by disturbance decoupled nonlinear observers, Proceedings of the CDC, Brighton, England, 1991, pp. 2248-2253.

[2] Hou, M. and Muller, P.C.: Fault detection and isolation observers, International Journal of Control, 1994, 60, (5), pp. 827-846.

[3] Gertler, J.: Fault detection and isolation using parity relations, Control Engineering Practice, 1997, 5, (5), pp. 653-661.

[4] Zhong, M., Ding, S. X., Lam J., and Wang, H.: An LMI approach to design robust fault detection filter for uncertain LTI systems, Automatica, 2003, 39, (3), pp. 543-550.

[5] Zhao, H., Zhong, M., Zhang, M.: $H_{\infty}$ fault detection for linear discrete time-varying systems with delayed state, IET Control Theory \& Applications, 2010, 4, (11), pp. 2303-2314.

[6] Wang, H. and Yang G.-H.: A finite frequency domain approach to fault detection for linear discrete-time systems, International Journal of Control, 2008, 81 (7), pp. 1162-1171.

[7] Yang, H., Xia, Y., and Liu, B.: Fault Detection for T-S Fuzzy Discrete Systems in Finite-Frequency Domain, IEEE Transactions on Systems, Man, and Cybernetics, 2011, 41, (4), pp. 911-920. 
[8] Long, Y. and Yang, G.-H.: Fault detection for a class of networked control systems with finite-frequency servo inputs and random packet dropouts, IET Control Theory \& Applications, 2012, 6(15): 2397-2408.

[9] Chen, J., and Patton, R. J.: Robust model-based fault diagnosis for dynamic systems (Kluwer Academic Publishers, Boston, 1999).

[10] Ding, S. X., Model-based fault diagnosis techniques: Design schemes, algorithms, and tools (Springer, Berlin, 2008).

[11] Isermann, R.: Fault-Diagnosis Systems: An Introduction from Fault Detection to Fault Tolerance, (Springer, Berlin, 2006).

[12] Frank, P. M.: Fault diagnosis in dynamic systems using analytical and knowledge based redundancyA survey and some new results, Automatica, 1990, 26, (3), pp. 459-474.

[13] Hwang, I., Kim, S., Kim, Y., Seah, C. E.: A survey of fault detection, isolation, and reconfiguration methods, IEEE Transactions on Control Systems Technology , 2010, 18, (3), pp. 636-653.

[14] Dai, X., Gao, Z.: From model, signal to knowledge: A data-driven perspective of fault detection and diagnosis, IEEE Transactions on Industrial Informatics, 2013, 9, (4), pp. 2226-2238.

[15] Dai, L.: Singular Control Systems (Springer, Berlin, 1989).

[16] Duan, G. R.: Analysis and design of descriptor linear systems (Springer, New York, 2010).

[17] Dai, L.: Observers for discrete singular systems, IEEE Transactions on Automatic Control, 1988, 33, (2), pp. 187-191.

[18] Darouach, M., and Boutayeb, M.: Design of observers for descriptor systems, IEEE Transactions on Automatic Control, 1995, 40, (7), pp. 1323-1327.

[19] Hou, M., and Muller, P. C.: Observer design for descriptor systems, IEEE Transactions on Automatic Control, 1999, 44, (1), pp. 164-169.

[20] Lu, G., and Ho, D. W. C.: Full-order and reduced-order observers for Lipschitz descriptor systems: The unified LMI approach, IEEE Transactions on Circuits and SystemsII: Express Briefs, 2006, 53, (7), pp. 563-567.

[21] Wang, Z., Shen, Y., Zhang, X., and Wang, Q.: Observer design for discrete-time descriptor systems: An LMI approach, Systems \& Control Letters, 2012, 61, (6), pp. 683-687.

[22] Hammouri, H., and Nadri, M.: An observer design for a class of implicit systems, Systems \& Control Letters, 2013, 62, (3), pp. 256-261.

[23] Vemuri, A. T., Polycarpou, M. M., and Ciric, A. R.: Fault diagnosis of differential-algebraic systems, IEEE Transactions on Systems, Man, and Cybernetics, Part A: Systems and Humans, 2001, 31, (2), pp. 143-152. 
[24] Duan, G. R., Howe, D., and Patton, R. J.: Robust fault detection in descriptor linear systems via generalized unknown input observers, International Journal of Systems Science, 2002, 33, (5), pp. 369-377.

[25] Gao, Z. and Ding, S. X.: Fault estimation and fault-tolerant control for descriptor systems via proportional, multiple-integral and derivative observer design, IET Control Theory \& Applications, 2007, 1, (5), pp. 1208-1218.

[26] Wang, Z., Shen, Y., and Zhang, X.: Actuator fault estimation for a class of nonlinear descriptor systems, International Journal of Systems Science, 2014, 45(3), pp. 487-496.

[27] Marx, B., Koenig, D., and Ragot, J.: Design of observers for Takagi-Sugeno descriptor systems with unknown inputs and application to fault diagnosis, IET Control Theory \& Applications, 2007, 1, (5), pp. 1487-1495.

[28] Chadli, M., Abdo, A., and Ding, S. X.: $H_{-} / H_{\infty}$ fault detection filter design for discrete-time Takagi-Sugeno fuzzy system, Automatica, 2013, 49, (7), pp. 1996-2005.

[29] Astorga-Zaragoza, C. M., Theilliol, D., Ponsart, J. C., and Rodrigues, M.: Fault diagnosis for a class of descriptor linear parameter-varying systems, International Journal of Adaptive Control and Signal Processing, 2012, 26, (3), pp. 208-223.

[30] Zhang, Y., and Jiang, J.: Bibliographical review on reconfigurable fault-tolerant control systems, Annual Reviews in Control, 2008, 32, (2), pp. 229-252.

[31] Zhong, M., Ding, S. X., Han, Q.-L., and Ding, Q.: Parity space-based fault estimation for linear discrete time-varying systems, IEEE Transactions on Automatic Control, 2010, 55, (7), pp. 1726-1731.

[32] Xu, A. and Zhang, Q.: Nonlinear system fault diagnosis based on adaptive estimation, Automatica, 2004, 40, (7), pp. 1181-1193.

[33] Zhang, K., Jiang, B., and Shi, P.: Fast fault estimation and accommodation for dynamical systems, IET Control Theory \& Applications, 2009, 3, (2), pp. 189-199.

[34] Shen, Y., Liu, L., and Dowell, E. H.: Actuator fault-tolerant robust control for a linear system with adaptive fault identification, IET Control Theory \& Applications, 2013, 7, (2), 246-252.

[35] Alwi, H., Edwards, C., and Tan, C. P.: Sliding mode estimation schemes for incipient sensor faults, Automatica, 2009, 45, (7), pp. 1679-1685.

[36] Jiang, B. and Chowdhury, F. N.: Fault estimation and accommodation for linear MIMO discrete-time systems, IEEE Transactions on Control Systems Technology, 2005, 13, (3), pp. 493-499.

[37] Keller, J.-Y.: Fault isolation filter design for linear stochastic systems, Automatica, 1999, 35, (10), pp. $1701-1706$.

[38] Ben-Israel, A. and Greville, T.: Generalized inverses: Theory and applications (Springer, New York, 2003, 2nd).

[39] Zhang, Q.: Adaptive observer for multiple-input-multiple-output (MIMO) linear time varying systems, IEEE 
Transactions on Automatic Control, 2002, 47, (3), pp. 525-529.

[40] Hsiung, K.-L. and Lee, L.: Lyapunov inequality and bounded real lemma for discrete-time descriptor systems, IEE Proceedings - Control Theory \& Applications, 1999, 146, (4), pp. 327-331.

[41] Boyd, S., Ghaoui, E., Feron, E., and Balakrishnan, V.: Linear matrix inequalities in system and control theory (Society for Industrial and Applied Mathematics, Philadelphia, 1994). 